\title{
Derivation of Capture Probabilities for the Corotation Eccentric Mean Motion Resonances
}

\author{
Maryame El Moutamid, ${ }^{1,2 \star}$ Bruno Sicardy, ${ }^{3}$ and Stéfan Renner ${ }^{4}$ \\ ${ }^{1}$ Cornell Center for Astrophysics, Planetary Science, Department of Astronomy, Cornell University, Ithaca, NY 14853, USA \\ ${ }^{2}$ Carl Sagan Institute, Cornell University, Ithaca, NY 14853, USA \\ ${ }^{3}$ LESIA, Observatoire de Paris, CNRS UMR 8109, Université Pierre et Marie Curie, Université Paris-Diderot, 5 place Jules Janssen, \\ F-92195 Meudon Cedex, France \\ ${ }^{4}$ IMCCE, Observatoire de Paris, CNRS UMR 8028, Université Lille 1, Observatoire de Lille 1 impasse de l'Observatoire, F-59000 Lille, France
}

4 September 2018

\begin{abstract}
We study in this paper the capture of a massless particle into an isolated, first order Corotation Eccentric Resonance (CER), in the framework of the Planar, Eccentric and Restricted Three-Body problem near a $m+1: m$ mean motion commensurability ( $m$ integer). While capture into Lindblad Eccentric Resonances (where the perturber's orbit is circular) has been investigated years ago, capture into CER (where the perturber's orbit is elliptic) has not yet been investigated in detail. Here, we derive the generic equations of motion near a CER in the general case where both the perturber and the test particle migrate. We derive the probability of capture in that context, and we examine more closely two particular cases: $(i)$ if only the perturber is migrating, capture is possible only if the migration is outward from the primary. Notably, the probability of capture is independent of the way the perturber migrates outward; $(i i)$ if only the test particle is migrating, then capture is possible only if the algebraic value of its migration rate is a decreasing function of orbital radius. In this case, the probability of capture is proportional to the radial gradient of migration. These results differ from the capture into Lindblad Eccentric Resonance (LER), where it is necessary that the orbits of the perturber and the test particle converge for capture to be possible. Possible applications for planetary satellites are discussed.
\end{abstract}

Key words: celestial mechanics - planets and satellites: dynamical evolution and stability - methods: analytical.

\section{INTRODUCTION}

Orbital captures into Mean Motion Resonance (MMR) is the key to understanding the orbital evolution of satellites, rings and planets. The special case of the Lindblad Eccentric Resonance (LER) has been investigated by many authors in the context of the Planar, Circular and Restricted ThreeBody Problem.

In this case, a secondary object orbiting a massive primary body perturbs a massless particle, so that the critical angle $\phi_{L}=(m+1) \lambda_{s}-m \lambda_{p}-\varpi_{p}$ librates, where $m$ is an integer, $\lambda_{s}$ and $\lambda_{p}$ are the longitudes of the secondary and the test particle, respectively, and $\varpi_{p}$ is the longitude of pericenter of the test particle. In a general context, Henrard (1982) estimated the probability of capture into a first order LER, while Borderies and Goldreich (1984) extended this

* E-mail: maryame@astro.cornell.edu work and derived capture probabilities into $m+1: m$ and $m+2: m$ (second order) LERs.

If the orbit of the secondary is eccentric, a Corotation Eccentric Resonance (CER) appears close to and associated with each LER. It is dynamically described by the critical angle $\phi_{c}=(m+1) \lambda_{s}-m \lambda_{p}-\varpi_{s}$, where $\varpi_{s}$ is the longitude of the secondary pericenter. The physical effects of LERs and CERs are not the same: the CER mainly affects the semi-major axis of the test particle and keeps its orbital eccentricity almost constant, forcing the particle to librate inside so-called corotation sites like a simple pendulum. In contrast, the LER acts on its eccentricity but keeps the semi-major axis almost constant (El Moutamid, Sicardy and Renner 2014).

In this work, we derive the probability of capturing a test particle into an isolated CER, as both the secondary and the particle suffer orbital migration that secularly change their semi-major axes. This is a novel calculation that com- 
plements what has been done before in the case of LERs. The term "isolated" means here that the CER and LER are sufficiently pulled apart so that their coupling is negligible. Eventually, the goal is to extend the study of captures into MMR to more realistic cases where both the CER and LER act in concert on the particle, but this will not be considered here.

This work can be applied to many situations, in the context of planetary rings and satellites. In the Saturnian system for example, the satellites Aegaeon, Anthe and Methone are respectively captured into 7:6, 10:11 and 14:15 CERs with Mimas (Cooper et al. 2008; Hedman et al. 2009, 2010; El Moutamid, Sicardy and Renner 2014). Atlas is in a 54:53 CER with Prometheus (Renner et al. 2016). In the case of the Neptunian system, Adams ring arcs may be dynamically confined by the satellite Galatea via corotation resonances (Renner et al. 2014; Nicholson et al. 1995; Foryta and Sicardy 1996; Sicardy et al. 1999).

We note here that the capture of a particle into a CER bears some resemblance with the capture of a rotating body into spin-orbit resonance (Goldreich and Peale 1966). In both cases, a slow effect (orbital migration in our case, tidal friction for the spin-orbit resonances) drives a pendulum-like system into a librating state, possibly capturing it permanently into that state. This will be commented later.

The paper is structured as follows: In section 2, we describe the dynamical structure of our problem based on the so-called CorALin model, taking into account the dissipation parameters. In Section 3, we study the particle and the secondary migration terms involved in the derivation of the probability of capture. The latter is derived in section 4 . In section 5 , we discuss the similarity of our problem with the capture in spin-orbit resonance and finally, a summary and conclusions are given in Section 6 .

\section{DYNAMICAL STRUCTURE OF THE PROBLEM}

We consider the restricted, planar (but not circular) threebody problem, in which a test particle orbits around a central mass $M_{c}$, near a first order mean motion resonance $m+1: m^{1}$ with a perturbing secondary of mass $m_{s}$ and an orbital eccentricity $e_{s}$. The various quantities and notations used hereafter are defined in Table 1.

Near the resonance, the equations of motion reduce to a two degree of freedom system associated with the two critical angles $\phi_{c}$ and $\phi_{L}$. The subscripts $c$ and $L$ refer to eccentric corotation and Lindblad resonances (CER and LER respectively). Details are given by El Moutamid, Sicardy and Renner (2014), who encapsulated the equations of motion of

1 The case $m$ positive (resp. negative) implies that the particle orbits inside (resp. outside) the secondary orbit.
Table 1. Variables, parameters and notations used in the text.

\begin{tabular}{cc}
\hline Quantities & Definitions \\
\hline$\phi_{c}$ & $(m+1) \lambda_{s}-m \lambda_{p}-\varpi_{s}$ \\
$\phi_{L}$ & $(m+1) \lambda_{s}-m \lambda_{p}-\varpi_{p}$ \\
$h$ & $\sqrt{3}|m| e_{p} \cos \left(\phi_{L}\right)$ \\
$k$ & $\sqrt{3}|m| e_{p} \sin \left(\phi_{L}\right)$ \\
$\chi$ & $3 m \Delta a / 2 a_{0}=3 m\left(a_{p}-a_{0}\right) / 2 a_{0}$ \\
$J_{c}$ & $\chi+\left(h^{2}+k^{2}\right) / 2$ \\
$\epsilon_{L}$ & $\sqrt{3}|m|\left(m_{s} / M_{c}\right)\left(a_{0} / a_{s}\right) A_{m}$ \\
$\epsilon_{c}$ & $3 m^{2}\left(m_{s} / M_{c}\right)\left(a_{0} / a_{s}\right) A_{m}^{\prime} e_{s}$ \\
$D$ & $\left(\dot{\varpi}_{s}-\dot{\varpi}_{p}\right) / n_{0}$ \\
\hline
\end{tabular}

Notes: $\lambda \overline{\varpi, a, e \text { denote the mean longitude, longitude of }}$ periapse, semi-major axis and eccentricity, respectively, and $\dot{\varpi}$ denotes the secular apsidal precession rate (e.g. forced by the oblateness of the central body or other perturbing secondaries). Subscripts $s$ and $p$ refer to the secondary and test particle, respectively. Subscript 0 is used for quantities estimated at the exact corotation radius, $a_{0}$. The quantities $A_{m}$ and $A_{m}^{\prime}$ are combinations of Laplace coefficients with $A_{m} \sim-A_{m}^{\prime} \sim 0.8 m$ for large $|m|$ 's. The quantities $\dot{\varpi}$ and $\dot{\varpi}_{s}$ are the rate of precession of the orbits of the test particle and the secondary, respectively.

the test particle in the so-called CorALin model ${ }^{2}$ :

$\left\{\begin{array}{l}d J_{c} / d \tau=-\epsilon_{c} \sin \left(\phi_{c}\right) \\ d \phi_{c} / d \tau=\chi \\ d h / d \tau=-(\chi+D) k \\ d k / d \tau=+(\chi+D) h+\epsilon_{L},\end{array}\right.$

where $\tau=n_{0} t$ is a dimentionless time scale, and $n_{0}$ is the mean motion at exact corotation ${ }^{3}$. The strengths of the corotation and Lindblad resonances are quantified by the parameters $\epsilon_{c}$ and $\epsilon_{L}$, respectively, see Table 1 .

The CorALin model portrays two coupled resonances: a CER described by a simple pendulum system (first two equations in Eqs. (1)), coupled to the LER given by the last two equations (the so-called second fundamental model for resonance). The dimensionless parameter $D$ measures the distance (in frequency) between the two resonances and acts as a coupling parameter between the two. For large $D$ 's, the resonances decouple and for $\chi=0$, only the simplependulum motion is relevant, while the particle eccentricity

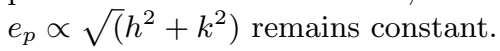

Stable oscillations of $\phi_{c}$ occur around $\phi_{c}=0$ (resp. $\phi_{c}=$ $\pi)$ for $\epsilon_{c}$ positive (resp. negative), with periods $\sim 2 \pi / \sqrt{\left|\epsilon_{c}\right|}$ in the CorALin time unit, or $\sim 2 \pi /\left(n_{0} \sqrt{\left|\epsilon_{c}\right|}\right)$ in the usual time unit. Moreover, the full width (in units of $\chi$ ) of the corotation site is $W=4 \sqrt{\left|\epsilon_{c}\right|}$, or

$W_{\mathrm{CER}}=\frac{8 a_{0} \sqrt{\left|\epsilon_{c}\right|}}{3|m|}$

2 The presentation of the CorALin model here is slightly different from the one given in El Moutamid, Sicardy and Renner (2014). The main differences are the sign of $\phi_{L}$ and the presence of the parameter $\epsilon_{c}$, which provides a better understanding of the physical effect of a corotation resonance.

3 Note that the time scale used later in this paper is the usual time $t$, and not $\tau$. 


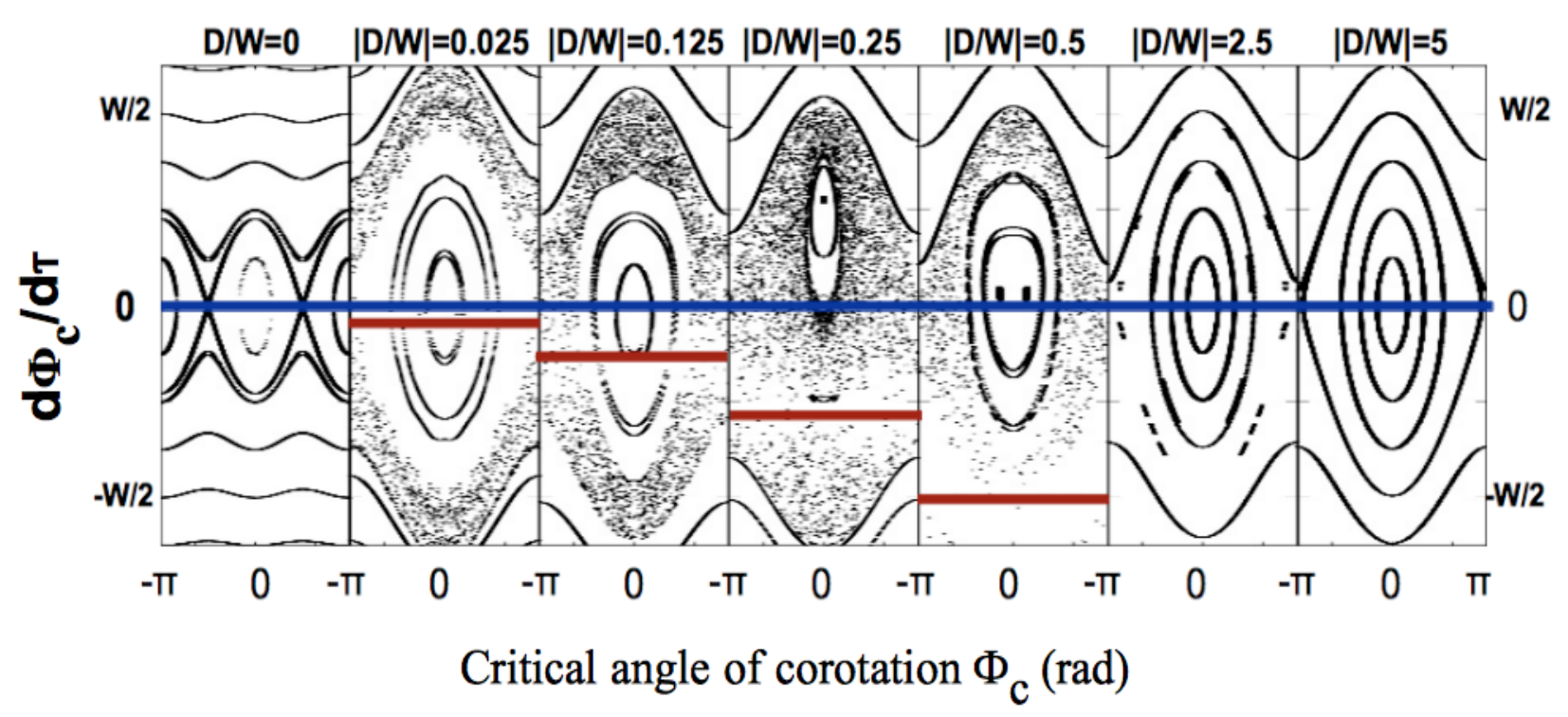

Figure 1. Poincaré surfaces of section of system (1) using $\epsilon_{L}=-0.1, \epsilon_{c}=1$ and $m=1$ (resonance 2:1) for seven different values of $D$. Each section is obtained when $k=0$ and $\dot{k}>0$ for 15 different initial conditions with the same value of energy. Note that $\tau=n_{0} t$ is a dimensionless time scale (more details are given in the text). For $D=0$ and large $D$ 's, the trajectories are regular, while chaos is dominant for intermediate cases, see more details in El Moutamid, Sicardy and Renner 2014. The blue lines represent the position of the center of CER, while the red lines represent the position of the center of the LER.

in physical distance units $^{4}$.

The decoupling between the CER and LER occurs for

$D_{\mathrm{CL}}>\sim W_{\mathrm{CER}}$,

where $D_{\mathrm{CL}}$ is the radial splitting between the resonances and given by:

$D_{\mathrm{CL}}=\frac{2 a_{0}}{3 m}\left(\frac{\dot{\varpi}_{s}-\dot{\varpi}_{p}}{n_{0}}\right)$,

The condition (3) is fulfilled in the right-most panels of Fig. (1). That figure shows that for intermediate values of $D_{\mathrm{CL}}$, the CER and LER are superimposed and strongly coupled, leading to chaotic behavior. However, the system is again an integrable one as $D_{\mathrm{CL}}=0$.

We will restrict our study to the case of large $D_{\mathrm{CL}}$ 's only (Eq. 3), and consider the effect of orbital migrations of both the secondary and the test particle. This causes a secular variation of $\chi$ (beyond the effect of the CER) since both $a_{p}$ and $a_{0}$ slowly change.

At this point, it is important to note that $\chi$ (a local form of the particle semi-major axis, see Table 1) is not the appropriate action variable to use together with the angle $\phi_{c}$. Instead, an angular momentum-type quantity should be used to avoid cumbersome additional terms in the equations of motion in the presence of migration, and permit the use of adiabatic invariance arguments. Here we choose the variable $J_{\phi}=3 m\left(J_{p}-J_{0}\right)$ as the conjugate of $\phi_{c}$, where $J_{p}$ is the specific angular momentum of the particle and $J_{0}=\sqrt{G M_{c} a_{0}}$ is the specific angular momentum at radius $a_{0}$ ( $G$ being the gravitational constant). As the CER has a very small effect on eccentricity in the absence of LER (El Moutamid, Sicardy

4 Note that $m$ and $\epsilon_{c}$ have opposite signs. and Renner 2014), we can assume here that the particle has a circular orbit, then $J_{\phi}=\chi J_{0}$ is proportional to $\chi$.

In the absence of migration, the Hamiltonian that locally describes the simple-pendulum motion near the CER reads:

$\mathcal{H}_{0}=\left(\frac{G M_{c}}{J_{0}}\right)^{2}\left[\frac{1}{2}\left(\frac{J_{\phi}}{J_{0}}\right)^{2}-\epsilon_{c} \cos \left(\phi_{c}\right)\right]$,

with the correct dimension of a specific energy (see Sicardy and Dubois (2003) for details on the method).

We now define $\epsilon_{s}$ (resp. $\epsilon_{p}$ ) as the dimensionless secular migration rates of the secondary (resp. test particle $)^{5}$ :

$$
\begin{aligned}
\epsilon_{s} & =\frac{1}{n_{0} a_{0}} \cdot \frac{d a_{0}}{d t}=\frac{2}{n_{0} J_{0}}\left(\frac{d J_{0}}{d t}\right)_{\mathrm{mig}} \\
\epsilon_{p} & =\frac{1}{n_{0} a_{0}} \cdot \frac{d a_{p}}{d t}=\frac{2}{n_{0} J_{0}}\left(\frac{d J_{p}}{d t}\right)_{\mathrm{mig}},
\end{aligned}
$$

where the "mig" index means the secular effect of the migration on the variation of $J_{0}$ and $J_{\phi}$.

Those migration rates introduce additional terms $\dot{J}_{\phi}=$ $(3 m / 2) n_{0} J_{0}\left(\epsilon_{p}-\epsilon_{s}\right)$ in the equations of motion ${ }^{6}$. They can be incorporated in $\mathcal{H}_{0}$ to form a new Hamiltonian:

$\mathcal{H}=\left(\frac{G M_{c}}{J_{0}}\right)^{2}\left[\frac{1}{2}\left(\frac{J_{\phi}}{J_{0}}\right)^{2}-\epsilon_{c} \cos \left(\phi_{c}\right)+\frac{3}{2} m \epsilon_{\operatorname{mig}} \phi_{c}\right]$,

\footnotetext{
5 Strictly speaking, $\epsilon_{s}$ actually measures the migration rate of the resonance radius $a_{0}$, related to the secondary migration rate by $d a_{0} / d t=[m /(m+1)]^{2 / 3}(d a / d t)$.

6 Note that $\epsilon_{c}$ does not vary during the migration of the secondary, due its mere definition (Table 1).
} 

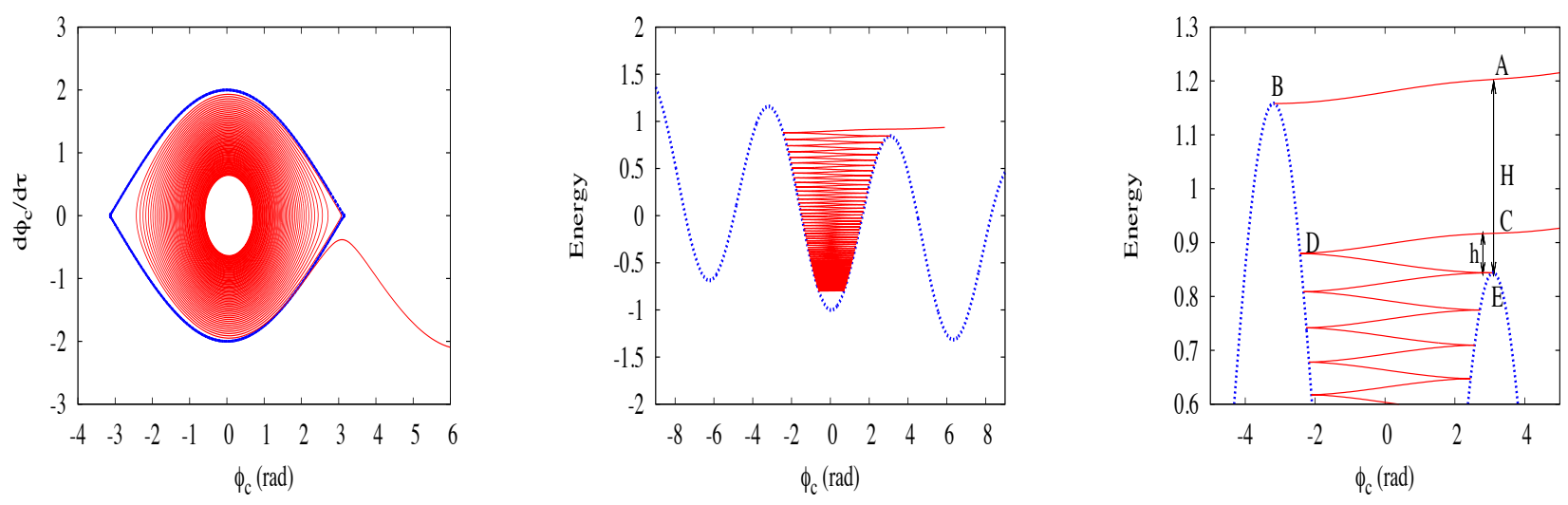

Figure 2. An example of a successful capture in CER, considering a case where $\epsilon_{c}>0$. The parameters $\left(\epsilon_{c}=1, \epsilon_{m i g}=5 \times 10^{-2}\right.$ and $d \epsilon_{m i g} / d \chi=-5 \times 10^{-3}$ ) have been greatly exaggerated (compared to practical cases) for better viewing. Note that $\tau=n_{0} t$ is a dimensionless time scale (more details are given in the text). Left panel: Phase space $\left(\phi_{c}, \dot{\phi}_{c}\right)$, where the blue curve represents the separatrix, and the red curve is the captured trajectory illustrated in the right panels. Middle panel- Blue curve: the potential energy $U$ (Eq. 11) versus $\phi_{c}$. Red curve: the total energy $E$ (Eq. 10). Right: vertical expansion of the middle panel, showing the parameters $h$ and $H$ used for the calculation of the probability of capture, see text.

where

$\epsilon_{\mathrm{mig}}=\epsilon_{s}-\epsilon_{p}$

Note the mirror-symmetry between the factors $\epsilon_{s}$ and $\epsilon_{p}$ : as expected, the migration of the secondary in one direction has the same effect as the migration of the particle in the opposite direction. Also, the Hamiltonian nature of the motion means that the particle cannot be captured in the CER: $\phi_{c}$ is either permanently librating or permanently circulating. This has a simple physical interpretation: the particle approaches the corotation radius at the same rate as it recedes from it. This symmetry prevents capture.

However, as $\epsilon_{s}$ is in general time-dependent and the particle migration is usually space-dependent, capture becomes possible. Here we make the simplest assumption that the particle migration has a local gradient parametrized by a dimensionless parameter $\epsilon_{g}$, that we define as follows:

$\frac{1}{n_{0} a_{0}} \frac{d a_{p}}{d t}=\epsilon_{p}+\epsilon_{g}\left(\frac{a_{p}-a_{0}}{a_{0}}\right)$.

As we see next, the factors $\epsilon_{s}$ and $\epsilon_{g}$ break the symmetry between the approach to and the recession from CER, possibly leading to capture. In the rest of the paper, all the terms involving the letter $\epsilon$ will be assumed to be small compared to unity, corresponding to the fact that the migration rates and their variations are assumed to be small.

\section{MIGRATION}

The shape of the particle trajectory in the $\left(J_{\phi}, \phi\right)$ phase space, in particular the fact that it is in libration or circulation, solely depends on the value of the term into brackets in Eq. (7):

$$
\begin{aligned}
E & =\frac{1}{2}\left[\frac{J_{\phi}}{J_{0}(t)}\right]^{2}-\epsilon_{c} \cos \left(\phi_{c}\right)+\frac{3}{2} m \epsilon_{\mathrm{mig}}(t) \phi_{c} \\
& =\frac{1}{2 n_{0}^{2}} \dot{\phi}_{c}^{2}-\epsilon_{c} \cos \left(\phi_{c}\right)+\frac{3}{2} m \epsilon_{\mathrm{mig}} \phi_{c}
\end{aligned}
$$

We have enhanced in the first equation that both $J_{0}$ and $\epsilon_{\text {mig }}$ explicitly depends upon time, due to migration, and we have used $\dot{\phi}_{c}=n_{0}\left(J_{\phi} / J_{0}\right)$ to write the second equation.

The quantity $E$ can be viewed as the dimensionless "energy" of a particle moving into the "potential" $U$ of a modified simple pendulum, where:

$U=-\epsilon_{c} \cos \left(\phi_{c}\right)+\frac{3}{2} m \epsilon_{\mathrm{mig}} \phi_{c}$.

The function $U\left(\phi_{c}\right)$ is plotted in blue in the two right-most panels of Fig. 2.

The two slowly varying parameters, $J_{0}$ and $\epsilon_{\mathrm{mig}}$, together with the term $\epsilon_{g}$, cause a variation of the energy $E$ :

$\dot{E}=\frac{d E}{d t}=-\frac{\epsilon n_{0}}{2}\left(\frac{J_{\phi}}{J_{0}}\right)^{2}+\frac{3}{2} m \dot{\epsilon}_{\mathrm{mig}} \phi_{c}=-\frac{\epsilon}{2 n_{0}} \dot{\phi}_{c}^{2}+\frac{3}{2} m \dot{\epsilon}_{\mathrm{mig}} \phi_{c}$

where $\epsilon=\epsilon_{s}-2 \epsilon_{g}$.

A necessary condition of capture is that the particle loses energy $E$, in order to converge toward a local minimum of $U$. Ignoring the term in $\dot{\epsilon}_{\text {mig }}$ (see Appendix), this requires:

$\epsilon=\epsilon_{s}-2 \epsilon_{g}>0$.

A paradoxical result is that $\epsilon$ depends on the migration rate $\epsilon_{s}$ of the secondary and on the gradient $\epsilon_{g}$ of the 
particle migration rate. In other words, there is a symmetry breaking between the respective effects of the secondary and the particle migrations. This stems from the fact that we consider here the restricted problem.

This effect is described Sicardy and Dubois (2003), who considered the different, but connected problem of two coorbital secondary masses $m_{1}$ and $m_{2}$ suffering slow migrations with rates $\epsilon_{1}$ and $\epsilon_{2}$, and gradients $G_{1}^{\prime}$ and $G_{2}^{\prime}$ (equivalent to the term $\epsilon_{g}$ used here), respectively. Their equation A.13 shows that $\dot{E}$ stems from two terms in front of $\dot{\phi}_{c}^{2}$, one proportional to $\left(m_{1} \epsilon_{1}+m_{2} \epsilon_{2}\right) /\left(m_{1}+m_{2}\right)$ and one proportional to $\left(m_{1} \epsilon_{2} G_{2}^{\prime}+m_{2} \epsilon_{1} G_{1}^{\prime}\right) /\left(m_{1}+m_{2}\right)$. As long as $m_{1}$ and $m_{2}$ are non-zero, there is a commutativity between the effects of the migrations of the two masses. As $m_{1}$, say, tends to zero, however one obtains a term in $\epsilon_{2}$ only (equivalent to the term in $\epsilon_{s}$ in Eq. 13) and a term in $\epsilon_{1} G_{1}^{\prime}$ only (equivalent to the term in $\epsilon_{g}$ ).

Turning back to Eq. (13), we see that if acting alone $\left(\epsilon_{g}=0\right)$, the secondary migration can lead to capture only if the migration is outwards $\left(\epsilon_{s}>0\right)$. If the particle migration is acting alone $\left(\epsilon_{s}=0\right)$, capture is possible only if its gradient is negative $\left(\epsilon_{g}<0\right)^{7}$. In that case, the particle approaches the CER faster than it recedes from it, permitting the capture.

Once the capture is effective, the particle will converge towards a local minimum of $U$, i.e. the CER center (Fig. 2). Considering the effect of secondary migration only, one can use an adiabatic invariant argument to estimate how the particle converges to that minimum. Using for instance $\epsilon_{c}>0$ and considering a motion close to the stable point $\phi_{c}=0$, we have $\cos \left(\phi_{c}\right) \sim 1-\phi_{c}^{2} / 2$, and thus a harmonic motion of the type $J_{\phi}=\mathbf{J}_{\phi} \cos (\omega t)$ and $\phi_{c}=\boldsymbol{\Phi}_{\mathbf{c}} \sin (\omega t)$, with $\omega=n_{0} \sqrt{\epsilon_{c}}$ and where boldfaces denote amplitudes. The equations $\dot{J}_{\phi}=-\partial \mathcal{H} / \partial \phi_{c}$ and $\dot{\phi}_{c}=+\partial \mathcal{H} / \partial J_{\phi}$ then provide $\mathbf{J}_{\phi}=\sqrt{\epsilon_{c}} J_{0} \boldsymbol{\Phi}_{\mathbf{c}}$, so that the action is $\mathcal{A}=\mathbf{J}_{\phi} \boldsymbol{\Phi}_{\mathbf{c}}=\sqrt{\epsilon_{c}} J_{0} \boldsymbol{\Phi}_{\mathbf{c}}^{\mathbf{2}}$, where $J_{0}=\sqrt{G M_{c} a_{0}}$. For a slow variation of $a_{0}, \mathcal{A}$ is adiabatically conserved so that $\sqrt{a_{0}} \boldsymbol{\Phi}_{\mathbf{c}}^{\mathbf{2}}=$ constant, thus:

$\Phi_{\mathbf{c}} \propto \frac{1}{a_{0}^{1 / 4}}$,

a result already obtained in the case of co-orbitals bodies by Fleming and Hamilton (2000) and Sicardy and Dubois (2003). Note that the convergence toward the CER center thus weakly depends upon $a_{0}$.

If the particle migration is acting alone, one obtains easily from the previous calculations that $d \boldsymbol{\Phi}_{\mathbf{c}} / d t=$ $\left(n_{0} \epsilon_{g} / 2\right) \boldsymbol{\Phi}_{\mathbf{c}}$, so that

$\boldsymbol{\Phi}_{\mathbf{c}} \propto \exp \left(n_{0} \epsilon_{g} t / 2\right)$,

where we recall that $\epsilon_{g}$ must be negative for capture to occur. Then the particle converges towards the CER center on a time scale $t \sim 2 /\left(n_{0}\left|\epsilon_{g}\right|\right)$.

When both the secondary and the particle migrate, the two equations above can be combined to provide:

$\boldsymbol{\Phi}_{\mathbf{c}} \propto \frac{\exp \left(\int \epsilon_{g} n_{0} d t / 2\right)}{a_{0}^{1 / 4}}$.

7 Note that this gradient concerns the algebraic value of the migration rate, not its absolute value. For instance an inward migration rate $\left(\epsilon_{p}<0\right)$ whose absolute value decreases with orbital radius will have a positive gradient $\left(\epsilon_{g}>0\right)$.
Note that the evaluations above can be used to estimate on which time scales the particle escapes the CER libration center for the cases $\epsilon<0$. This may happen if the particle is formed inside the CER site, e.g. a dust particle ejected from the surface of a body currently trapped in a CER.

\section{PROBABILITY OF CAPTURE}

Fig. 2 illustrates the capture mechanism. It closely follows the path described in Goldreich and Peale (1966) and Murray and Dermott (1999) for capture in spin-orbit resonances. The right-most panel shows that the particle must approach the corotation site through a small energy "window" $h$, inside the possible interval $H$. The particle moves in that plot along the red curve with slope:

$\frac{d E}{d \phi_{c}}=\frac{1}{\dot{\phi}_{c}} \frac{d E}{d t}=-\frac{\epsilon}{2 n_{0}} \dot{\phi}_{c}$,

where we have neglected the term in $\dot{\epsilon}_{\text {mig }}$ in Eq. (12), see Appendix.

The trajectories $\mathrm{AB}$ and $\mathrm{CDE}$ bound the extreme trajectories that will escape the CER. As those trajectories come very near the hyperbolic points at $\phi_{c} \approx \pi$ and $-\pi$, they have energy $E \approx+\epsilon_{c}$, so that $\dot{\phi}_{c} \approx$ $\pm n_{0} \sqrt{2\left|\epsilon_{c}\right|\left(1+\cos \left(\phi_{c}\right)\right)}= \pm 2 n_{0} \sqrt{\left|\epsilon_{c}\right|} \cos \left(\phi_{c} / 2\right)$. Injecting that expression into Eq. (17), one can integrate $d E / d \phi_{c}$ to obtain the variations of $E$ alongs the trajectories $\mathrm{AB}$ and CDE. Elementary calculations based on the form of $U$ (Eq. 11) then provide:

$h=8 \epsilon \sqrt{\left|\epsilon_{c}\right|}$ and $H=3 \pi\left|m \epsilon_{\mathrm{mig}}\right|+4 \epsilon \sqrt{\left|\epsilon_{c}\right|}$,

from which the probability of capture $P_{m}=h / H$ into the $m+1: m$ CER is derived, assuming that the initial energy of the particle is randomly and uniformly distributed in the "window" $H$. Substituting the value of $\epsilon_{c}$ as given in Eq. (2) we obtain:

$P_{m}=\frac{h}{H}=\frac{2 \epsilon W_{\mathrm{CER}}}{2 \pi a_{0}\left|\epsilon_{\mathrm{mig}}\right|+\epsilon W_{\mathrm{CER}}}$,

where we recall that $\epsilon=\epsilon_{s}-2 \epsilon_{g}$ and $\epsilon_{\mathrm{mig}}=\epsilon_{s}-\epsilon_{p}$. We also recall that the condition $\epsilon>0$ must be fulfilled for the capture to occur (Eq. 13).

At this point, we note that in general $W_{\text {CER }} \ll a_{0}$, since $\epsilon_{c}$ is very small in Eq. 2, as $m_{s} / M_{c}$ and $e_{s}$ are themselves usually small (Table 1). Moreover, in general, the particle migration rate $d a_{p} / d t$ does not change rapidly with distance. Taking for instance $d a_{p} / d t=K / a_{p}^{q}$, where $K$ is a constant and $q$ is usually of order unity, we obtain from Eq. (9) $\epsilon_{g}=$ $-q \epsilon_{p}$. As a consequence $\epsilon_{g}$ and $\epsilon_{p}$ are usually of same order, so that the term in $W_{\mathrm{CER}}$ in the denominator of the equation above can be neglected. Thus, the probability of capture when both the secondary and the particle migrate is:

$P_{m} \approx \frac{W_{\mathrm{CER}}}{\pi a_{0}}\left|\frac{\epsilon}{\epsilon_{\mathrm{mig}}}\right|=\frac{W_{\mathrm{CER}}}{\pi a_{0}}\left|\frac{\epsilon_{s}-2 \epsilon_{g}}{\epsilon_{s}-\epsilon_{p}}\right|$.

We now examine the two extreme cases where $(i)$ only the particle migrates and where (ii) only the secondary mi- 
grates. In case $(i)$, we have $\epsilon_{s}=0$, so that

$$
\begin{array}{ll}
P_{m} \approx \frac{2 W_{\mathrm{CER}}}{\pi a_{0}}\left|\frac{\epsilon_{g}}{\epsilon_{p}}\right|= & \\
\frac{2 W_{\mathrm{CER}}}{\pi a_{\epsilon p}} \quad \text { (particle migration only) }
\end{array}
$$

where we define $a_{\epsilon p}=a_{0}\left|\epsilon_{p} / \epsilon_{g}\right|$ as the radial scale over which the particle migration rate suffers significant changes. We recall here that the condition $\epsilon_{g}<0$ must be fulfilled.

In case $(i i)$, we have $\epsilon_{p}=\epsilon_{g}=0$ (and $\left.\epsilon_{s}>0\right)$, so that

$P_{m} \approx \frac{W_{\mathrm{CER}}}{\pi a_{0}} \quad$ (secondary migration only)

with the noteworthy result that in this case the probability of capture is independent of the way the secondary migrates. In other words, we do not need to know the details of that migration to derive the probability of capture. Note also from Eq. (2) and Eq. (22) that $P_{m}$ is independent of $a_{0}$, since $\epsilon_{c}$ is a constant. In fact, $P_{m}$ now only depends on $\epsilon_{c}$ and $m$ (i.e. the secondary mass and orbital eccentricity, plus some geometrical factors, see Table 1).

Eq. (22) can be re-written by noting that the physical area enclosed in each corotation site (see the physical area enclosed in the blue separatrix in the left-most panel of Fig. 2) is $4 a_{0} W_{\mathrm{CER}} /|m|$. Since there are $|m|$ such corotation sites, the total area occupied by the CER is $S_{\mathrm{CER}}=4 a_{0} W_{\mathrm{CER}}$. Moreover, the area enclosed of an orbit of semi-major axis $a_{0}$ is $S_{0}=\pi a_{0}^{2}$, so that:

$P_{m} \approx \frac{S_{\mathrm{CER}}}{4 S_{0}}$.

Thus, if only the secondary migrates, the probability of capture is one quarter of the area occupied by the CER libration sites divided by the area enclosed in the CER orbit.

\section{SIMILARITIES WITH SPIN-ORBIT RESONANCES}

At this stage, we note that the capture of a particle into a CER resembles the capture of a rotating body into spin-orbit resonance (see Goldreich and Peale 1966 or Murray and Dermott 1999). This follows from the fact that the dynamical structures of CERs and spin-orbit resonances are both based on simple pendulum systems. The capture happens because of orbital migration in the case of a CER, and tidal friction (and thus migration in the frequency space) for spin-orbit resonances. Using Eq. 2 and the fact that the libration frequency around the corotation center is $\omega_{0}=n_{0} \sqrt{\left|\epsilon_{c}\right|}$, one can re-write eq. 19 as:

$P_{m}=\frac{4\left(\omega_{0} / n_{0}\right)}{(3 / 4) \pi a_{0}|m|\left|\epsilon_{\mathrm{mig}} / \epsilon\right|+2\left(\omega_{0} / n_{0}\right)}$.

Murray and Dermott (1999) provide the probability of capture into a spin-orbit resonance $\Omega=p n_{0}$ (where $\Omega$ is the spin rate of the body and $p$ is a rational) in two different cases. One of them corresponds to a tidal torque that is dependent on the frequency of each tidal component (the frequency in this context means the one with which the body experiences the tidal distortion), $P_{p}=4\left(\omega_{0} / n_{0}\right) /\left[\pi V+2\left(\omega_{0} / n_{0}\right)\right]$, see their equation (5.118). The factor $V$ enters in expression of the average, frequency-dependent tidal torque acting on the body $\left\langle N_{s}\right\rangle=-K\left(V+\dot{\gamma} / n_{0}\right)$, where $K$ is a constant and $\gamma=\theta-p M$ is the spin-orbit resonant angle that relates the body orientation in inertial space, $\theta$, and its mean anomaly, $M$. By writing the tidal torque $\left\langle N_{s}\right\rangle=N_{s 0}+N_{s g}\left(\dot{\gamma} / n_{0}\right)$, where $N_{s 0}$ is the local tidal torque and $N_{s g}$ is its local gradient (in the frequency space), we obtain $V=N_{s 0} / N_{s g}$. Therefore, $P_{p}$ can be written:

$P_{p}=\frac{4\left(\omega_{0} / n\right)}{\pi\left(N_{s 0} / N_{s g}\right)+2\left(\omega_{0} / n\right)}$,

identical to Eq. 24 after posing $N_{s 0} / N_{s g}=(3|m| / 2)\left|\epsilon_{\text {mig }} / \epsilon\right|$. In the special case where $\epsilon_{s}=0$ (i.e. when only the particle migrates), we obtain $N_{s 0} / N_{s g}=(3|m| / 4)\left|\epsilon_{p} / \epsilon_{g}\right|$, which enhances the essential similarities between the CER and spinorbit captures. Both describe a particle that migrates along the frequency axis of the simple-pendulum phase space at rate $N_{s 0}$, with a local gradient $N_{s g}$ along that axis.

\section{DISCUSSIONS AND CONCLUSIONS}

We have studied the mechanism of capture into an isolated first order Corotation Eccentric Resonance (CER) in the context of the Elliptical Restricted Three-body Problem, in which a test particle orbits around a central mass $M_{c}$, near a first order mean motion resonance $m+1: m$ with a perturbing secondary of mass $m_{s}$. We derive a formula for the probability of capture given by Eq. 20 in a general context. Then we apply this result to two particular cases, first where we consider a migration of the particle only (Eq. 21), and secondly, in the case of migration of the secondary (Eq. 22). We point out the noteworthy fact that in this case, the capture probability does not depend on the way the secondary migrates.

Under realistic assumptions, we rewrite our formula for capture in the second case as Eq. (23). This equation has the interesting consequence that if the corotation radius $a_{0}$ sweeps a total area $S$ in a region where particles are uniformly distributed with surface density $N$, then the corotation sites will eventually be populated (due to captures) with a particle surface density of $N S / 4 S_{0}$. Conversely, if we observe today a certain number of particles trapped in corotation sites, we may estimate the number of particles originally present in that region.

This work can be applied in many cases, specially in the context of planetary rings, satellites and in the context of exoplanets. As an example, in the context of the Saturn system, El Moutamid, Sicardy and Renner (2014) study the case of Aegaeon which is trapped in a 7:6 CER caused by Mimas, with $a_{0} \sim 167,500 \mathrm{~km}$. Since $W_{\mathrm{CER}} \sim 30 \mathrm{~km}$, we find a probability of capture of $\sim 5 \times 10^{-5}$. This very low probability suggests that originally, there were many more such objects in the Saturn system, but that only a few of them were captured into CERs.

Note finally that this study assumes that the CER is isolated, i.e. that the condition (3) is fulfilled. In the cases where $D_{\mathrm{CL}} \sim W_{\mathrm{CER}}$, the motion in phase space is chaotic, as the LER and CER are strongly coupled, see Fig. 1. This occurs when the effects of the oblateness of the central body, the presence of a massive disk or another companion in the 
system, are not strong enough to split and thus isolate the resonances from each other. The chaotic nature of motion then prevents an easy analytical derivation of the probability of capture into the $m+1: m$ mean motion resonance. In that case, numerical integrations might be useful to see whether the probability derived here (Eq. 19) remains valid, at least in order of magnitude.

\section{ACKNOWLEDGEMENTS}

The authors are thankful to Philip D. Nicholson, Aurélien Crida, Damya Souami and Matthew M. Hedman for many interesting discussions on this topic. They thank the Encelade working group for interesting discussions and the International Space Science Institute (ISSI) for support. This work was supported by NASA through the Cassini project. Part of the research leading to these results has received funding from the European Research Council under the European Community's H2020 (2014-2020/ ERC Grant Agreement no 669416 "LUCKY STAR").

\section{REFERENCES}

Borderies, N. and Goldreich, P., 1984, Celestial Mechanics, 32, 127-136

Charnoz, S., Crida, A., Castillo-Rogez, J. C., Lainey, V., Dones, L., Karatekin, Ö., Tobie, G., Mathis, S., Le Poncin-Lafitte, C. and Salmon, J., 2011, Icarus, 216, 535-550

Cooper, N. J., Murray, C. D., Evans, M. W., Beurle, K., Jacobson, R. A. and Porco, C. C., 2008, Icarus, 195, 765-777

Crida, A. and Charnoz, S., 2012, Science, 338, 1196

El Moutamid M., Sicardy, B. and Renner, S., 2014, Celestial Mechanics and Dynamical Astronomy, 118, 235-252

Fleming, H. J. and Hamilton, D. P., 2000, Icarus, 148, 479-493

Foryta, D. W. and Sicardy, B., 1996, Icarus, 123, 129-167

Goldreich, P. and Peale, S., 1966, AJ, 71, 425

Hedman, M. M., Murray, C. D., Cooper, N. J., Tiscareno, M. S., Beurle, K., Evans, M. W. and Burns, J. A. , 2009, Icarus, 199, 378-386

Hedman, M. M., Cooper, N. J., Murray, C. D., Beurle, K., Evans, M. W., Tiscareno, M. S. and Burns, J. A., 2010, Icarus, 207, 433-447

Henrard, J., 1982, Celestial Mechanics, 27, 3-22

Murray, C. D. and Dermott, S. F., 1999, Solar system dynamics book

Nicholson, P. D., Mosqueira, I. and Matthews, K., 1995, Icarus, 113, 295-330

Renner, S., Sicardy, B., Souami, D., Carry, B. and Dumas, C., 2014, A\&A, 563, A133

Renner, S., Cooper, N. J., El Moutamid, M., Sicardy, B., Vienne, A., Murray, C. D. and Saillenfest, M., 2016, AJ, 151, 122

Sicardy, B., Roddier, F., Roddier, C., Perozzi, E., Graves, J. E., Guyon, O. and Northcott, M. J., 1999, Nature, 400, 731-733

Sicardy, B. and Dubois, V., 2013, Celestial Mechanics and Dynamical Astronomy, 86, 321-350

\section{APPENDIX A: EFFECT OF THE VARIATION OF MIGRATION RATE WITH TIME}

If the secondary migrates, and since that migration usually depends on the parameter $a_{0}, \epsilon_{s}$ also depends on time, as well as $\epsilon_{m i g}=\epsilon_{s}-\epsilon_{p}$. In that case, the term $(3 m / 2) \dot{\epsilon}_{m i g} \phi_{c}=$
$(3 m / 2) \dot{\epsilon}_{s} \phi_{c}$ in Eq. (12) causes a variation of $E$ that should be compared to $-\epsilon \dot{\phi}_{c}^{2} / 2 n_{0}=-2 n_{0} \epsilon\left|\epsilon_{c}\right| \cos ^{2}\left(\phi_{c} / 2\right)$. In order to simplify the analysis, we assume a simple functional form $d a_{0} / d t=K / a_{0}^{q}$, as is done after Eq. (19). Thus, $\dot{\epsilon}_{s}=-(q-$ $1 / 2) n_{0} \epsilon_{s}^{2}$.

Consequently, in order to neglect $(3 m / 2) \dot{\epsilon}_{m} \phi_{c}$ with respect to $2 n_{0} \epsilon\left|\epsilon_{c}\right| \cos ^{2}\left(\phi_{c} / 2\right)$, we must have $|m|(q-$ $1 / 2)\left(\epsilon_{s} / \epsilon_{c}\right) \ll 1$. Defining the orbital migration time scale as $t_{\text {mig }}=a_{0} / \dot{a}_{0}$, denoting $T_{0}$ the orbital period $\left(2 \pi / n_{0}\right)$, and using Eq. (2), the condition above reads

$\frac{q-1 / 2}{|m|}\left(\frac{a_{0}}{W_{\mathrm{CER}}}\right)^{2} T_{0} \ll t_{m}$.

Equivalently, this requires that the radial velocity $v_{\text {mig }}=d a_{0} / d t$ resulting from the migration of the secondary fulfills the condition:

$v_{\text {mig }} \ll \frac{|m|}{2 \pi(q-1 / 2)}\left(\frac{W_{\mathrm{CER}}}{a_{0}}\right)^{2} v_{0}$,

where $v_{0}=2 \pi a_{0} / T_{0}$ is the orbital velocity.

Those conditions must be checked on a case-by-case basis. For instance, El Moutamid, Sicardy and Renner (2014) study the case of Aegaeon which is trapped in a 7:6 CER caused by Mimas, with $m=6, a_{0} \sim 167,500 \mathrm{~km}, W_{\mathrm{CER}} \sim$ $50 \mathrm{~km}$ and $T_{0} \sim 0.81$ days. If we consider a Mimas migration caused by tides raised on Saturn, then $q=11 / 2$ (Murray and Dermott 1999). The condition (A1) thus requires $t_{m} \gg 20,000$ years. This is satisfied by a safe margin, as typical migration time scales for Mimas are of the order of several millions years (Charnoz et al. 2011; Crida and Charnoz 2012).

This paper has been typeset from a $\mathrm{T}_{\mathrm{E}} \mathrm{X} / \mathrm{L} \mathrm{T}_{\mathrm{E}} \mathrm{X}$ file prepared by the author. 